\title{
LEAVITT PATH ALGEBRAS OF FINITE GELFAND-KIRILLOV DIMENSION
}

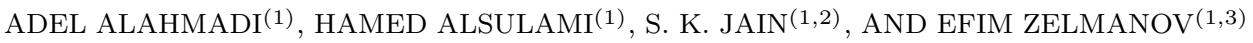

\begin{abstract}
Groebner-Shirshov basis and Gelfand-Kirillov dimension of the Leavitt path algebra are derived.
\end{abstract}

\section{Introduction.}

Leavitt path algebras were introduced in $[\mathrm{AA}]$ as algebraic analogs of graph Cuntz-Kreiger $\mathrm{C}^{*}$-algebras. Since then they have received significant attention from algebraists. In this paper we (i) find a Groebner-Shirshov basis of a Leavitt path algebra, (ii) determine necessary and sufficient conditions for polynomially bounded growth, and (iii) find Gelfand-Kirillov dimension.

\section{Definitions and Terminologies}

A (directed) graph $\Gamma=(V, E, s, r)$ consists of two sets $V$ and $E$, called vertices and edges respectively, and two maps $s, r: E \rightarrow V$. The vertices $s(e)$ and $r(e)$ are referred to as the source and the range of the edge $e$, respectively. The graph is called row-finite if for all vertices $v \in V,\left|\left(s^{-1}(v)\right)\right|<\infty$. A vertex $v$ for which $\left(s^{-1}(v)\right)$ is empty is called a sink. A path $p=e_{1} \ldots e_{n}$ in a graph $\Gamma$ is a sequence of edges $e_{1} \ldots e_{n}$ such that $r\left(e_{i}\right)=s\left(e_{i+1}\right)$ for $i=1, \ldots,(n-1)$. In this case we say that the path $p$ starts at the vertex $s\left(e_{1}\right)$ and ends at the vertex $r\left(e_{n}\right)$. If $s\left(e_{1}\right)=r\left(e_{n}\right)$, then the path is closed. If $p=e_{1} \ldots e_{n}$ is a closed path and the vertices $s\left(e_{1}\right), . ., s\left(e_{n}\right)$ are distinct, then the subgraph $\left(s\left(e_{1}\right), \ldots, s\left(e_{n}\right) ; e_{1}, \ldots, e_{n}\right)$ of the graph $\Gamma$ is called a cycle.

Let $\Gamma$ be a row-finite graph and let $F$ be a field. The Leavitt path $F$-algebra $L(\Gamma)$ is the $F$-algebra presented by the set of generators $\{v, v \in V\},\left\{e, e^{*} \mid e \in E\right\}$ and the set of relators (1) $v_{i} v_{j}=\delta_{v_{i}, v_{j}} v_{i}$ for all $v_{i}, v_{j} \in V ;(2) s(e) e=e r(e)=e$, $r(e) e^{*}=e^{*} s(e)=e^{*}$, for all $e \in E ;(3) e^{*} f=\delta_{e, f} r(e)$, for all $e, f \in E$; (4) $v=\sum_{s(e)=v} e e^{*}$, for an arbitrary vertex $v \in V \backslash\{\operatorname{sinks}\}$.

The mapping which sends $v$ to $v$, for $v \in V$, e to $e^{*}$ and $e^{*}$ to $e$, for $e \in E$, extends to an involution of the algebra $L(\Gamma)$. If $p=e_{1} \ldots e_{n}$ is a path, then $p^{*}=e_{n}^{*} \ldots e_{1}^{*}$.

\section{A Basis OF $L(\Gamma)$}

For an arbitrary vertex $v$ which is not a sink, choose an edge $\gamma(v)$ such that $s(\gamma(v))=v$. We will refer to this edge as special. In other words, we fix a function $\gamma: V \backslash\{\operatorname{sinks}\} \rightarrow E$ such that $s(\gamma(v))=v$ for an arbitrary $v \in V \backslash\{$ sinks $\}$.

Key words and phrases. Leavitt Path Algebra. Cuntz-Krieger C*-Algebras. GroebnerShirshov Basis. Polynomially Bounded Growth. Gelfand-Kirillov Dimension. 
Theorem 1. The following elements form a basis of the Leavitt path algebra $L(\Gamma)$ :

(i) $v, v \in V$, (ii) $p, p^{*}$, where $p$ is a path in $\Gamma$, (iii) $p q^{*}$, where $p=e_{1} \ldots e_{n}$, $q=f_{1} \ldots f_{m}, e_{i}, f_{j} \in E$, are paths that end at the same vertex $r\left(e_{n}\right)=r\left(f_{m}\right)$, with the condition that the last edges $e_{n}$ and $f_{m}$ are either distinct or equal, but not special.

Proof. Recall that a well-ordering on a set is a total order (that is, any two elements can be ordered) such that every non-empty subset of elements has a least element.

As a first step, we will introduce a certain well-ordering on the set of generators $X=V \cup E \cup E^{*}$. Choose an arbitrary well-ordering on the set of vertices $V$. If $e, f$ are edges and $s(e)<s(f)$ then $e<f$. It remains to order edges that have the same source. Let $v$ be a vertex which is not a sink. Let $e_{1}, \ldots, e_{k}$ be all the edges that originate from $v$. Suppose $e_{k}=\gamma(v)$. We order the edges as follows: $e_{1}<e_{2}<\ldots .<e_{k}=\gamma(v)$. Choose an arbitrary well-ordering on the set $E^{*}$. For arbitrary elements $v \in V, e \in E, f^{*} \in E^{*}$, we let $v<e<f^{*}$. Thus the set $X=V \cup E \cup E^{*}$ is well-ordered. Let $X^{*}$ be the set of all words in the alphabet $X$. The length-lex order (see [B, Be]) makes $X^{*}$ a well-ordered set. For all $v \in V$ and $e \in E$, we extend the set of relators (1) - (4) by (5): ve $=0$, for $v \neq s(e) ; e v=0$, for $v \neq r(e) ; v e^{*}=0$, for $v \neq r(e) ; e^{*} v=0$, for $v \neq s(e)$. The straightforward computations show that the set of relators (1) - (5) is closed with respect to compositions (see $[\mathrm{B}, \mathrm{BE}]$ ). By the Composition-Diamond Lemma ([B, $\mathrm{BE}]$ ) the set of irreducible words (not containing the leading monomials of relators (1) - (5) as subwords) is a basis of $L(\Gamma)$. This completes the proof.

\section{LEAVITT PATH ALGEBRAS OF POLYNOMIAL GROWTH}

Recall some general facts on the growth of algebras. Let $A$ be an algebra (not necessarily unital), which is generated by a finite dimensional subspace $V$. Let $V^{k}$ denote the span of all products $v_{1} \cdots v_{k}, v_{i} \in V, k \leq n$. Then $V=V^{1} \subset$ $V^{2} \subset \cdots, A=\cup_{n \geq 1} V^{n}$ and $g_{V(n)}=\operatorname{dim} V^{n}<\infty$. Given the functions $f, g$ from $N=\{1,2, \ldots\}$ to the positive real numbers $R_{+}$, we say that $f \preccurlyeq g$ if there exists $c \in N$ such that $f(n) \leq c g(c n)$ for all $n$. If $f \preccurlyeq g$ and $g \preccurlyeq f$ then the functions $f, g$ are said to be asymptotically equivalent, and we write $f \sim g$. If $W$ is another finite dimensional subspace that generates $A$, then $g_{V(n)} \sim g_{W(n)}$. If $g_{V(n)}$ is polynomially bounded, then we define the Gelfand-Kirillov dimension of $A$ as $G K \operatorname{dim} A=\lim \sup _{n \rightarrow \infty} \frac{\ln g_{V(n)}}{\ln n}$. The definition of $G K$-dimension does not depend on a choice of the generating space $V$ as long as $\operatorname{dim} V<\infty$. If the growth of $A$ is not polynomially bounded, then $G K \operatorname{dim} A=\infty$.

We now focus on finitely generated algebras and we will assume that the graph $\Gamma$ is finite. Let $C_{1}, C_{2}$ be distinct cycles such that $V\left(C_{1}\right) \cap V\left(C_{2}\right) \neq \phi$. Then we can renumber the vertices so that $C_{1}=\left(v_{1}, \cdots, v_{m} ; e_{1}, \cdots, e_{m}\right), C_{2}=\left(w_{1}, \cdots, w_{n} ; f_{1}, \cdots\right.$ $\left.\cdot, f_{n}\right), v_{1}=w_{1}$. Let $p=e_{1} \cdots e_{m}$, and $q=f_{1} \cdots f_{n}$.

Lemma 2. The elements $p, q$ generate a free subalgebra in $L(\Gamma)$.

Proof. By Theorem 1, different paths viewed as elements of $L(\Gamma)$ are linearly independent. If $u_{1}, u_{2}$ are different words in two variables, then $u_{1}(p, q)$ and $u_{2}(p, q)$ are different paths. Indeed, cutting out a possible common beginning, we can assume that $u_{1}, u_{2}$ start with different letters, say, $u_{1}(p, q)=p \cdot \cdot, u_{2}(p, q)=q \cdots$. If 
$m>n$ then the path $u_{2}(p, q)$ returns to the vertex $v_{1}$ at the $n$-th step, whereas $u_{1}(p, q)$ does not. If $m=n$, then the left segments of length $m$ of $u_{1}(p, q), u_{2}(p, q)$ are different. This proves the lemma.

Corollary 3. If two distinct cycles have a common vertex, then $L(\Gamma)$ has exponential growth.

From now on we will assume that any two distinct cycles of the graph $\Gamma$ do not have a common vertex.

For two cycles $C^{\prime}, C^{\prime \prime}$, we write $C^{\prime} \Longrightarrow C^{\prime \prime}$, if there exists a path that starts in $C^{\prime}$ and ends in $C^{\prime \prime}$.

Lemma 4. If $C^{\prime}, C^{\prime \prime}$ are two cycles such that $C^{\prime} \Longrightarrow C^{\prime \prime}$, and $C^{\prime \prime} \Longrightarrow C^{\prime}$, then $C^{\prime}=C^{\prime \prime}$.

Proof. Choose a path $p$ that starts in $C^{\prime}$ and ends in $C^{\prime \prime}$. Similarly, choose a path $q$ that starts in $C^{\prime \prime}$ and finishes in $C^{\prime}$. There exists also a path $p^{\prime}$ on $C^{\prime \prime}$, which connects $r(p)$ with $s(q)$ and a path $q^{\prime}$ on $C^{\prime}$, which connects $r(q)$ with $s(p)$. Now, $p p^{\prime} q q^{\prime}$ is a closed path, which visits both $C^{\prime}$ and $C^{\prime \prime}$. Let $t$ be a closed path with this property (visiting both $C^{\prime}$ and $C^{\prime \prime}$ ) having a minimal length. Write $t=$ $e_{1} \cdots e_{n}, e_{i} \in E$. We claim that the vertices $s\left(e_{1}\right), \cdots, s\left(e_{n}\right)$ are all distinct, thus $t=\left(s\left(e_{1}\right), \cdots, s\left(e_{n}\right) ; e_{1}, \cdots, e_{n}\right)$ is a cycle. Assuming the contrary, let $s\left(e_{i}\right)=s\left(e_{j}\right)$, $1 \leq i<j \leq n$, and $j-i$ is minimal with this property. Then $t^{\prime}=\left(s\left(e_{i}\right), s\left(e_{i+1}\right), \cdots\right.$ .,$\left.s\left(e_{j}\right) ; e_{i}, e_{i+1}, \cdots, e_{j-1}\right)$ is a cycle. Let us "cut it out", that is, consider the path $t^{\prime \prime}=e_{1} \cdots e_{i-1} e_{j} \cdots e_{n}$. This path is shorter than $t$. Hence $t^{\prime \prime}$ can not visit both $C^{\prime}$ and $C^{\prime \prime}$. Suppose that $t^{\prime \prime}$ does not visit $C^{\prime}$. Then at least one of the vertices $s\left(e_{i}\right), \cdots, s\left(e_{j-1}\right)$ lies in $C^{\prime}$. Since two intersecting cycles coincide, it implies that $t^{\prime}=C^{\prime}$, hence $s\left(e_{j}\right)$ lies in $C^{\prime}$. This contradicts our assumption that $t^{\prime \prime}$ does not visit $C^{\prime}$. Hence $t=C^{\prime}=C^{\prime \prime}$. This proves the lemma.

A sequence of distinct cycles $C_{1}, \cdots, C_{k}$ is a chain of length $k$ if $C_{1} \Longrightarrow \cdots \Longrightarrow$ $C_{k}$. The chain is said to have an exit if the cycle $C_{k}$ has an exit (see [AA]), that is, if there exists an edge $e$ such that $s(e) \in V\left(C_{k}\right)$, but $e$ does not belong to $C_{k}$. Let $d_{1}$ be the maximal length of a chain of cycles in $\Gamma$, and let $d_{2}$ be the maximal length of chain of cycles with an exit. Clearly, $d_{2} \leq d_{1}$.

Theorem 5. Let $\Gamma$ be a finite graph.

(1) The Leavitt path algebra $L(\Gamma)$ has polynomially bounded growth if and only if any two distinct cycles of $\Gamma$ do not have a common vertex;

(2) If $d_{1}$ is the maximal length of a chain of cycles in $\Gamma$, and $d_{2}$ is the maximal length of chain of cycles with an exit, then $G K \operatorname{dim} L(\Gamma)=\max \left(2 d_{1}-1,2 d_{2}\right)$.

Proof. As in the proof of Theorem 1 we consider the generating set $X=V \cup E \cup E^{*}$ of $L(\Gamma)$. Let $E^{\prime}$ be the set of edges that do not belong to any cycle. Let $P^{\prime}$ be the set of all paths that are composed from edges from $E^{\prime}$. Then an arbitrary path from $P^{\prime}$ never arrives to the same vertex twice. Hence, $\left|P^{\prime}\right|<\infty$.

By Theorem 1 the space $\operatorname{Span}\left(X^{n}\right)$ is spanned by elements of the following types:

(1) a vertex,

(2) a path $p=p_{1}^{\prime} p_{1} p_{2}^{\prime} p_{2} \cdots p_{k} p_{k+1}^{\prime}$, where $p_{i}$ is a path on a cycle $C_{i}, 1 \leq i \leq k$, $C_{1} \Longrightarrow \cdots \Longrightarrow C_{k}$ is a chain, $p_{i}^{\prime} \in P^{\prime}$, length $(p) \leq n$,

(3) $p^{*}$, where $p$ is a path of the type (2), 
(4) $p q^{*}$, where $p=p_{1}^{\prime} p_{1} p_{2}^{\prime} p_{2} \cdots p_{k} p_{k+1}^{\prime}, q=q_{1}^{\prime} q_{1} q_{2}^{\prime} \cdots q_{s} q_{s+1}^{\prime} ; p_{i}, q_{j}$ are paths on cycles $C_{i}, D_{j}$ respectively and $C_{1} \Longrightarrow \cdots \Longrightarrow C_{k}, D_{1} \Longrightarrow \cdots \Longrightarrow D s$ are chains ; $p_{i}^{\prime}, q_{j}^{\prime} \in P^{\prime}$, length $(p)+$ length $(q) \leq n$ with $r(p)=r(q)$. We will further subdivide this case into two subcases:

(4.1) $r(p) \notin V\left(C_{k}\right) \cup V\left(D_{s}\right)$;

(4.2) $r(p) \in V\left(C_{k}\right) \cup V\left(D_{s}\right)$.

We will estimate the number of products of length $\leq n$ in each of the above cases and then use the following elementary fact:

Let $\left(a_{n}\right)_{n \in N}$ be the sum of $s$ sequences $\left(a_{i n}\right)_{n \in N}, 1 \leq i \leq s, a_{i n}>0$. Then

$\lim \sup _{n \rightarrow \infty} \frac{\ln a_{n}}{\ln n}=\max \left(\lim \sup _{n \rightarrow \infty} \frac{\ln a_{i n}}{\ln n}, 1 \leq i \leq s\right)$

Let us estimate the number of paths of the type (2). Fix a chain $C_{1} \Longrightarrow C_{2}$ $\Longrightarrow \ldots \Longrightarrow C_{k}$. If $C=\left(v_{1}, \ldots, v_{m} ; e_{1}, \ldots, e_{m}\right)$ is a cycle, let $P_{C}=e_{1} \ldots e_{m}$. For a given cycle there are $m$ such paths depending upon the choice of the starting point $v_{1}$.

Let $\mid\left(V\left(C_{i}\right) \mid=m_{i}\right.$ and let $P_{C_{i}}$ be any one of the $m_{i}$ paths described above. Then an arbitrary path on $C_{i}$ can be represented as $u^{\prime} P_{i}^{l} u^{\prime \prime}$, where length $\left(u^{\prime}\right)$, length $\left(u^{\prime \prime}\right) \leq m_{i}-1$. Hence every path of the type (2) which corresponds to the chain $C_{1}$ $\Longrightarrow C_{2} \Longrightarrow \ldots \Longrightarrow C_{k}$ can be represented as $p_{1}^{\prime} u_{1}^{\prime} P_{C_{1}}^{l_{1}} u_{1}^{\prime \prime} \ldots p_{k}^{\prime} u_{k}^{\prime} P_{C k}^{l_{k}} u_{k}^{\prime \prime} p_{k+1}^{\prime}$, where $p_{i}^{\prime} \in P_{i}^{\prime}$ and length $\left(u_{i}^{\prime}\right)$, length $\left(u_{i}^{\prime \prime}\right) \leq m_{i}-1$. Clearly, $m_{1} l_{1}+\ldots+m_{k} l_{k} \leq n$. This implies that the number of such paths $\preccurlyeq n^{k} \leq n^{d_{1}}$. On the other hand, choosing a chain $C_{1} \Longrightarrow C_{2} \Longrightarrow \ldots \Longrightarrow C_{d_{1}}$ of length $\bar{d}_{1}$, we can construct $\sim n^{d_{1}}$ paths of length $\leq n$. The case (3) is similar to the case (2).

Consider now the elements of length $\leq n$ of the type $p q^{*}, r(p)=r(q)$; the path $p$ passes through the cycles of the chain $C_{1} \Longrightarrow C_{2} \Longrightarrow \ldots \Longrightarrow C_{k}$ on the way, the path $q$ passes through the cycles of the chain $D_{1} \Longrightarrow D_{2} \Longrightarrow \ldots \Longrightarrow D_{s}$ on the way and so $p=p_{1}^{\prime} p_{1} p_{2}^{\prime} \ldots p_{k} p_{k+1}^{\prime}$, where $p_{i}^{\prime} \in P_{i}^{\prime}$, each $p_{i}$ is a path on the cycle $C_{i}$. Similarly, $q=q_{1}^{\prime} q_{1} q_{2}^{\prime} \ldots q_{s} q_{s+1}^{\prime}$. Arguing as above, we see that for fixed chains $C_{1}$ $\Longrightarrow C_{2} \Longrightarrow \ldots \Longrightarrow C_{k}$ and $D_{1} \Longrightarrow D_{2} \Longrightarrow \ldots \Longrightarrow D_{s}$, the number of such paths $\preccurlyeq n^{k+s}$.

Suppose that the vertex $v=r(p)=r(q)$ does not lie in $V\left(C_{k}\right) \cup V\left(D_{s}\right)$. Then both cycles $C_{k}$ and $D_{s}$ have exits. Hence the number of paths of type (4.1) is $\leq n^{2 d_{2}}$. On the other hand, let $C_{1} \Longrightarrow C_{2} \Longrightarrow \ldots \Longrightarrow C_{d_{2}}$ be a chain and let $e$ be an exit of the cycle $C_{d_{2}}$. Select paths $p_{2}^{\prime}, \ldots, p_{d_{2}}^{\prime}$, where $p_{i}^{\prime}$ connects $C_{i-1}$ to $C_{i}$, $p_{i}^{\prime} \in P^{\prime}$.

Select a path $u_{1}^{\prime \prime}$ on the cycle $C_{1}$ which connects $r\left(P_{C_{1}}\right)$ to $s\left(p_{2}^{\prime}\right)$, a path $u_{2}^{\prime}$ in $C_{2}$ which connects $r\left(p_{2}^{\prime}\right)$ to $s\left(P_{C_{2}}\right)$, a path $u_{2}^{\prime \prime}$ on $C_{2}$ which connects $r\left(P_{C_{2}}\right)$ to $s\left(P_{3}^{\prime}\right)$, and so on. The path $u_{d_{2}}^{\prime \prime}$ connects $r\left(P_{C_{d_{2}}}\right)$ to $s(e)$.

Among the edges from $s^{-1}(s(e))$ choose a special one $\gamma(s(e))$ different from $e$. Then by Theorem 1 , the elements

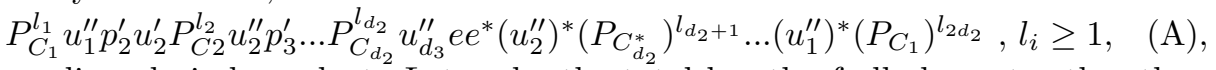

are linearly independent. Let $m$ be the total length of all elements other than $P_{C{ }_{i}}^{l_{i}},\left(P_{C_{i}^{*}}\right)^{l_{2 d_{2}-i+1}}$. The number of elements in the inequality $(\mathrm{A})$ above is the number of nonnegative integral solutions of the inequality

$\sum_{i=1}^{d_{2}} m_{i}\left(l_{i}+l_{2 d_{2}-i+1}\right) \leq n-m$, which is $\sim n^{2 d_{2}}$.

Now suppose that the vertex $v=r(p)=r(q)$ lies in $C_{k}$. Assume at first that $C_{k} \neq D_{s}$. Then the chain $D_{1} \Longrightarrow D_{2} \Longrightarrow \ldots \Longrightarrow D_{s}$ has an exit. If $k \leq s$, then the number of the paths of this type is $\leq n^{k+s} \leq n^{2 d_{2}}$. 
If $s<k$, then $n^{k+s} \leq n^{2 k-1} \leq n^{2 d_{1}}-1$.

Next, let $C_{k}=D_{s}$. It means that the paths $p_{k+1}^{\prime}, q_{s+1}^{\prime}$ are empty; $p_{k}$ and $q_{s}$ are both paths on the cycle $C_{k}$ and in this case we have,

(i) $p_{k} q_{s}^{*}=u$, if $p_{k}=u q_{s}$, is a path on $C_{k}$,

(ii) $p_{k} q_{s}^{*}=u^{*}$, if $q_{s}=u p_{k}$, is a path on $C_{k}$, and

(iii) $p_{k} q_{s}^{*}=0$, otherwise.

The number of such elements $p q^{*}$ is $\preccurlyeq n^{k+s-1} \leq n^{2 d_{1}}-1$.

On the other hand, let $C_{1} \Longrightarrow C_{2} \Longrightarrow \ldots \Longrightarrow C_{d_{2}}$ be a chain of cycles. Select paths $p_{2}^{\prime}, \ldots, p_{d_{1}}^{\prime} \in P^{\prime}, p_{i}^{\prime}$ connects $C_{i-1}$ to $C_{i} ; u_{i}^{\prime}, u_{i}^{\prime \prime}$ are paths on the cycle $C_{i}$ such that $P_{C_{1}} u_{1}^{\prime \prime} p_{2}^{\prime} u_{2}^{\prime} P_{C_{2}} u_{2}^{\prime \prime} p_{3}^{\prime} \ldots P_{C_{d_{1}}} \neq 0$. By Theorem 1, the elements $P_{C_{1}}^{l_{1}} u_{1}^{\prime \prime} p_{2}^{\prime} u_{2}^{\prime} P_{C_{2}}^{l_{2}} u_{2}^{\prime \prime} p_{3}^{\prime} \ldots u_{d_{1}}^{\prime} P_{C_{d_{1}}}^{l_{d_{1}}}\left(u_{d_{1}}^{\prime}\right)^{*}\left(P_{C_{d_{1}-1}}^{*}\right)^{l_{d_{1}+1}} \ldots\left(P_{C_{1}}^{*}\right)^{l_{2 d_{1}-1}}$ are linearly independent provided that $l_{i} \geq 1,1 \leq i \leq 2 d_{1}-1$. The number of these elements is $\sim$ $n^{2 d_{1}-1}$. This proves Theorem 2 .

References.

[AA] G. Abrams, G. Aranda Pino, The Leavitt path algebra of a graph, J. Algebra 293 (2005), 319-334

[B] L.A. Bokut, Imbeddings in simple associative algebras, Algebra i Logica, 15 (1976), 117-142.

[Be] G.M. Bergman, The Diamond Lemma for ring theory, Adv. in Math. 29 (1978), 178-218.

[KL] G.R.Krause, T.H. Lenagan, Growth of Algebras and Gelfand-Kinillow Dimension, revised edition. Graduate studies in Mathematics, 22, AMS, Providence, RI, 2000

1. Department of Mathematics, King Abdulaziz University, Jeddah, SA, 2.Department of Mathematics, Ohio University, USA, 3 Department of Mathematicsw, University of California, San Diego, Partially supported by the NSF 